\title{
Challenges with Dissolution Testing and Quality Assessment for Commercial Feverfew Products
}

\author{
Ping Jin', Shadi Madieh², and Larry L. Augsburger ${ }^{3}$ \\ 'United States Pharmacopeia, 12601 Twinbrook Parkway, Rockville, Maryland 20852, USA. \\ ${ }^{2}$ Glaxo Smith Kline, 709 Swedeland Road, King of Prussia, PA 19406, USA. \\ ${ }^{3}$ University of Maryland, Baltimore, School of Pharmacy, 20 N. Pine Street, Baltimore, MD 21201, \\ USA.
}

\begin{abstract}
The purpose of this study was to develop a suitable dissolution test for feverfew products and to investigate the parthenolide content, disintegration, and dissolution performance of several marketed feverfew products. Parthenolide content was determined by a validated HPLC method. Disintegration was tested according to the USP requirements for botanical dosage forms. A suitable dissolution test method was developed to study the dissolution performance of feverfew products.

Parthenolide content varied from 0 to $0.23 \%$ among the feverfew products tested. The average disintegration times of these products were significantly different $(p<0.005)$ and varied from 5 to $11 \mathrm{~min}$. All products containing measurable parthenolide exhibited more than $85 \%$ dissolution of parthenolide content in $1 \mathrm{~h}$; however, these products exhibited substantially different dissolution profiles. In the first $10 \mathrm{~min}$, one product released more than $80 \%$, but dissolution from the two other products could not be detected at all during that time. Although not currently required by USP, dissolution testing of formulated products can be a useful tool to evaluate the quality of feverfew finished products. Patients should be cautious when changing from one brand to another because of such differences in actual parthenolide content and release performance. Manufacturers should commit to proper quality control procedures to ensure that label claims for content and dose are accurate and realistic.
\end{abstract}

\section{Introduction}

he need for quality assurance, including content uniformity, compliance with the label strength, and appropriate and consistent release properties, has long been recognized for drug products containing chemically defined, synthetically produced drugs. However, for botanical products, this is not necessarily the case. In the United States, most botanical products are introduced into the market as dietary supplements. In the USP general chapter for dietary supplements, microbial limit, weight variation, and disintegration tests are specifically defined for botanical dosage forms. However, dissolution tests are currently available in the USP for four botanicals only: Ginger, Garlic Delayed Release, Milk Thistle, and Ginkgo, and compliance with the dissolution standard is optional for manufacturers of these products (1). In 2007, the FDA issued a final rule regarding current Good Manufacturing Practices for dietary supplements, which does not address any specific requirements for dissolution or disintegration (2).

The lack of a dissolution standard may be partly due to the chemical complexity of botanicals. All botanicals are complex mixtures containing multiple chemical components, and often there are several classes of compounds in a single product. The pharmacological effect of botanicals may be from a single component or the combination of multiple compounds. The ratio of different compounds may also affect the efficacy of the product. So far, even for some of the most popular botanicals, little is known about their pharmacological or toxicological profiles. All of these limitations cause great difficulty in identifying the individual components that can represent the pharmacological activity and be used for the evaluation of release properties of botanical products.

Different countries have different regulatory guidelines for dissolution testing of botanical products. Since 2000, the European Pharmacopoeia has introduced three classes of plant extracts based on knowledge of the composition of the herbal medicinal products and how well the relationship of composition to activity is known. These are "standardized" extracts, "quantified" extracts, and "other" extracts (3). Standardized extracts are herbal preparations whose active moiety has been definitely identified and adjusted to a given content; examples include Senna and Belladonna extracts. Quantified extracts refer to those products in which there is a defined, realistic range of constituent(s) that are accepted to contribute to activity; examples include Ginkgo and St. John's wort extracts. Other extracts are products for which no individual active component has been identified, for example, Extr. Valerianae and Extr. Echinaceae. Generally, botanical products made from quantified extracts and other extracts need not undergo dissolution testing as long as they are formulated as immediate-release products. For 
botanical products made from standardized extracts, dissolution testing is required in Germany. However, European Medicinal Evaluation Agency (EMEA) proposed that a disintegration test may substitute for a dissolution test if the active ingredient is known to be highly soluble in aqueous solution at $\mathrm{pH}$ values typical of the gastrointestinal tract (4).

Clearly, one could argue that whenever the components that contribute to the activity have been identified, dissolution testing can and should contribute to the product quality evaluation. This means that dissolution testing would be appropriate for botanical products made from standardized extracts and quantified extracts. Feverfew is a typical example of a quantified extract. As a botanical that has a long history of use in Europe, feverfew has become more and more popular in America and is ranked among the top twenty selling herbs in North America (5). Feverfew is believed to be useful in preventing migraine headaches and treating rheumatoid arthritis. Like most botanicals, feverfew is quite complex chemically and contains several potentially active components, such as the sesquiterpene lactones, flavonoid glycosides, sesquiterpenes, and monoterpenes. However, only parthenolide, the most abundant sesquiterpene lactone, is currently considered to be the primary active component. The effect of other components has not yet been determined (6).

In monographs of feverfew, the most important standard for quality control is the level of parthenolide (not less than $0.2 \%$ in USA, UK, and Canada, and $0.1 \%$ in France) (6). Daily doses of feverfew are not clearly defined in USP; however, the feverfew monograph in Canada suggests a daily dose of 50-250 mg feverfew dried leaf containing at least $0.2 \%$ parthenolide and not exceeding the equivalent of $4 \mathrm{mg}$ parthenolide per day (7).

To date, research on the quality of feverfew products has been focused on their parthenolide content. The wide variation among brands and large differences between actual content and label claims of parthenolide in feverfew have been frequently reported in published papers (8-10). However, there are no reports on the disintegration and dissolution performance of feverfew products. The objective of this study is to develop a suitable dissolution test for feverfew products and then use it as part of a comparative evaluation of the quality of several commercial feverfew products.

\section{Reagents and Samples}

Parthenolide ( $97 \%$ purity) was purchased from EMD Biosciences, Inc. (San Diego, CA). Five feverfew products were purchased from pharmacies in the Baltimore area. Their characteristics are described in Table 1. One powdered feverfew extract (Amax, Eugene, OR) was used for the development of the dissolution method.

HPLC-grade acetonitrile (Burdick \& Jackson, Muskegon, MI); potassium phosphate, monobasic (Sigma Chemical Co., St. Louis, MO); reagent-grade phosphoric acid 85\% (J.T. Baker, Phillipsburg, NJ); and HPLC-grade alcohol and methanol (E.M. Science, Gibbstown, NJ) were used. All water was purified using an in-house Milli-Q system (Millipore, Milford, MA).

\section{Methods \\ Sample Preparation for Parthenolide Content Assay}

Ten capsules of each product were weighed individually before and after emptying the capsule shell to calculate the weight of feverfew content (total weight-shell weight). The contents of these 10 capsules were combined and ground with a mortar and pestle until homogenous. An accurately weighed amount $(250 \mathrm{mg}$ ) of the powder was added to a $25-\mathrm{mL}$ volumetric flask, and $20 \mathrm{~mL}$ of $50 \%$ ethanol was added. After the mixture was sonicated for $30 \mathrm{~min}$ at room temperature, the final volume was brought to $25 \mathrm{~mL}$ with $50 \%$ ethanol. An appropriate volume $(\approx 2 \mathrm{~mL}$ ) was passed through a $0.45-\mu \mathrm{m}$ PTFE filter. The first milliliter was discarded, and the remaining filtrate was collected in a brown sample vial for HPLC analysis. The extraction procedure mentioned above and the following HPLC analytical conditions were developed and validated previously in our laboratory (11).

\section{Table 1. Characteristics of the Feverfew Products Studied Based on Label Claims.}

\begin{tabular}{|c|c|c|c|c|}
\hline Product & Dosage Form & $\begin{array}{l}\text { Feverfew extract/unit } \\
\text { (\% parthenolide in extract) }\end{array}$ & Other Ingredients & Suggested Use (Unit/d) \\
\hline P1 & Capsule & $250 \mathrm{mg}(0.7 \%)$ & Feverfew leaf $130 \mathrm{mg}$ & 1 \\
\hline $\mathrm{P} 2$ & Capsule (Vegetarian) & $250 \mathrm{mg}(0.7 \%)$ & Rice flour, cellulose, magnesium stearate & 2 \\
\hline P3 & Capsule (Vegetarian) & $200 \mathrm{mg}(0.7 \%)$ & $\begin{array}{l}\text { Feverfew aerial part } 150 \mathrm{mg} \text {, cellulose, magnesium } \\
\text { stearate, Vitamin C, Vitamin E, citric acid, rosemary extract }\end{array}$ & $1-3$ \\
\hline P4 & Capsule & $625 \mathrm{mg}(0.2 \%)$ & None & 4 \\
\hline P5 & Capsule & $250 \mathrm{mg}(0.7 \%)$ & Microcrystalline cellulose, silica & 1 \\
\hline
\end{tabular}




\section{HPLC Analysis of Parthenolide in Feverfew}

The HPLC system was equipped with a Hitachi L-7100 pump, L-7250 auto-sampler, and L-7400 UV detector. Separation was accomplished on a Gemini C18 column, $150 \times 4.6 \mathrm{~mm}$ i.d., $5-\mu \mathrm{m}$ particle size with a Security Guard cartridge system (Phenomenex, Torrance, CA).

Chromatographic data were processed with D-7000 HPLC system manager software (Merck/Hitachi). Quantitation was performed using parthenolide as an external standard. All injections were performed in triplicate unless otherwise stated.

The mobile phase, consisting of solvent A $(10 \mathrm{mM}$ $\mathrm{NaH}_{2} \mathrm{PO}_{4}$ in $\mathrm{H}_{2} \mathrm{O}$, adjusted to $\mathrm{pH} 3.0$ with phosphoric acid) and solvent $\mathrm{B}$ (acetonitrile), was degassed with helium. The flow rate was $1.5 \mathrm{~mL} / \mathrm{min}$ with a linear gradient as in Table 2, and UV detection was carried out at $210 \mathrm{~nm}$. The column temperature was maintained at $25^{\circ} \mathrm{C}$ during all determinations.

For dissolution testing, the method was validated over the range of $0.36-9 \mu \mathrm{g} / \mathrm{mL}$ parthenolide, and the injection volume was $30 \mu \mathrm{L}$. For general sample assay, the method was validated over $3.6-90 \mu \mathrm{g} / \mathrm{mL}$ parthenolide, and the injection volume was $10 \mu \mathrm{L}$.

\section{Dissolution Medium Selection and Optimization}

A single feverfew extract was selected for evaluation. To determine the effect of $\mathrm{pH}$ on the dissolution of parthenolide, $1.5 \mathrm{~g}$ of feverfew was placed in several 15-mL centrifuge tubes, and $4 \mathrm{~mL}$ of $0.1 \mathrm{M}$ buffer $(\mathrm{pH} 3,5$, and 7) was added to each tube. The tubes were shaken for $24 \mathrm{~h}$ at $37^{\circ} \mathrm{C}$, and then centrifuged at $3000 \mathrm{rpm}$ for $5 \mathrm{~min}$. The filtered supernatant was taken for HPLC analysis.

To determine the effect of $\mathrm{pH}$ on the extraction efficiency of parthenolide, samples were extracted as described in sample preparation above, except that buffers with different $\mathrm{pH}$ values $(3,5$, and 7$)$ replaced $50 \%$ ethanol as the extraction solvent.

In order to optimize the concentration of sodium dodecyl sulfate (SDS) used in dissolution testing, different

Table 2. Elution Time Profile of HPLC Method for the Determination of Parthenolide in Feverfew.

\begin{tabular}{lccl}
\hline Time $(\mathrm{min})$ & Solution A (\%) & Solution B (\%) & Elution \\
\hline $0-7.5$ & 59 & 41 & isocratic \\
\hline $7.5-10$ & $59 \rightarrow 30$ & $41 \rightarrow 70$ & linear gradient \\
\hline $10-15$ & 30 & 70 & isocratic \\
\hline $15-17$ & $30 \rightarrow 59$ & $70 \rightarrow 41$ & linear gradient \\
\hline $17-21$ & 59 & 41 & isocratic \\
\hline
\end{tabular}

amounts of SDS $(0.5 \%, 1 \%$, and $2 \%)$ were added to water as an extraction solvent. All other procedures were the same as described above in sample preparation.

\section{Dissolution Testing}

Dissolution testing was conducted using USP Method I (basket apparatus) (VanKel VK7000, VanKel Industries, Edison, NJ) at a temperature of $37 \pm 0.5^{\circ} \mathrm{C}$. Hydrodynamic conditions were standardized at $100 \mathrm{rpm}$ and $500 \mathrm{~mL}$ medium for each test. For each product under each dissolution condition, three tests were run in parallel, one capsule in each vessel in each test. Samples $(2 \mathrm{~mL})$ were withdrawn at 5, 10, 20,30,45,60, and 120 min and replaced immediately with prewarmed medium. Samples were filtered with a $0.45-\mu \mathrm{m}$ PTFE filter as mentioned above for HPLC analysis.

\section{Disintegration Testing}

The apparatus complied with the USP standard. One capsule and a disk were placed in each of six tubes of the basket assembly using $0.05 \mathrm{M}$ acetate buffer $(\mathrm{pH} 4.5 \pm 0.05)$ maintained at $37 \pm 2{ }^{\circ} \mathrm{C}$ as the immersion fluid. The disintegration time for each capsule was recorded. Capsules were considered completely disintegrated when only fragments of the capsule shell were left in the basket.

\section{Statistical Analysis}

Where appropriate, Student $t$-test or analysis of variance (ANOVA) was used to compare the data. The data were considered to be significant when $p<0.05$.

\section{Results and Discussion \\ Parthenolide Content of Five Feverfew Products}

As shown in Table 1, feverfew products are present in the market mainly as capsules. Feverfew powdered crude parts, excipients, or other botanical extracts may be combined into a feverfew formulation. Results shown in Table 3 demonstrate that most of the products cannot reach the $0.2 \%$ USP minimum requirement. However, current USP monographs for feverfew only apply to feverfew raw material and powdered raw material (1), and the presence of any additional excipients may be expected to lower the overall parthenolide percentage in the finished product. Because all products stated the amount of standardized parthenolide extract used, we calculated the claimed parthenolide content and compared that with the actual parthenolide content. It was found that actual parthenolide contents were far less than their label claims. One product even contained no detectable parthenolide. This product was excluded from the following dissolution study. 
Table 3. Parthenolide Content and Daily Dose of Commercial Feverfew Products.

\begin{tabular}{|c|c|c|c|c|c|}
\hline \multirow[b]{2}{*}{ Product } & \multirow[b]{2}{*}{ Unit Net Weight $^{1}(\mathrm{mg})$} & \multicolumn{4}{|c|}{ Parthenolide } \\
\hline & & $\begin{array}{l}\text { Claimed Unit } \\
\text { Strength }(\mu g)\end{array}$ & $\begin{array}{c}\text { Assayed } \\
\text { Parthenolide }^{2}(\%)\end{array}$ & $\begin{array}{c}\text { Actual Unit } \\
\text { Strength }^{3}(\mu g)\end{array}$ & $\begin{array}{l}\text { Actual Daily } \\
\text { Dose }^{4}(\mu \mathrm{g} / \mathrm{d})\end{array}$ \\
\hline P1 & $421.4 \pm 17.9$ & 1750 & $0.228 \pm 0.001$ & 961 & 961 \\
\hline P2 & $470.0 \pm 36.0$ & 1750 & $0.173 \pm 0.001$ & 813 & 1626 \\
\hline P3 & $383.1 \pm 5.6$ & 1050 & $0.124 \pm 0.001$ & 475 & $475-1425$ \\
\hline P4 & $646.5 \pm 10.7$ & 1250 & $0.054 \pm 0.000$ & 349 & 1397 \\
\hline P5 & $450.0 \pm 2.6$ & 1750 & 0 & 0 & 0 \\
\hline
\end{tabular}

${ }^{1}$ Content weight in each capsule (Total capsule weight - shell weight), $n=10$

${ }^{2}$ Percent parthenolide of capsule content, $n=3$

${ }^{3}$ Actual parthenolide content in each capsule in $\mu \mathrm{g}(\% \times$ content weight $\times 1000)$

${ }^{4}$ Daily dose based on actual unit strength and recommended unit/day

The dosage instructions on product labels vary from one to four units daily. According to product label claims, the suggested daily dose of feverfew can provide $1050-5000 \mu \mathrm{g}$ parthenolide. One product, $\mathrm{P} 4$, suggests a daily dose equivalent to $5000 \mu \mathrm{g}$ parthenolide, which exceeds the maximum daily dose recommended by the Canadian feverfew monograph. The actual daily doses of parthenolide calculated from the assays varied from 0 to $\approx 1600 \mu \mathrm{g}$ among the products, which indicates that in spite of the low parthenolide content of feverfew products in the current market, most of them still provide the required minimum amount of parthenolide.

\section{Dissolution Testing Specification Development}

In vitro dissolution specifications are established to ensure batch-to-batch consistency and to signal potential problems with in vivo bioavailability. The setting of dissolution specifications for a new chemical entity should be based on a consideration of the biopharmaceutical characteristic of the drug substance such as $\mathrm{pH}$ solubility profile, $\mathrm{pK}_{\mathrm{a}}$ and permeability (12). The Biopharmaceutics Classification System (BCS) category of a drug, which includes both solubility and permeability, can be used to predict the likelihood of a successful in vitro-in vivo correlation (IVIVC) (13). A good IVIVC is most likely available for low solubility-high permeability drugs. According to Khan et al. (14), parthenolide can be effectively absorbed through the intestinal mucosa via a passive diffusion mechanism.

The $\mathrm{pH}$-solubility profile is also directly related to the selection of the dissolution medium. It is desirable that the volume of the dissolution medium be sufficient to ensure a reasonable approximation of sink conditions, often assumed if the test volume is 5-10 times the saturated volume (15). Based on our data (not included here), parthenolide is very unstable under extreme acidic or basic conditions. Thus, a pH range of 3-7 was selected to determine the effect of $\mathrm{pH}$ on the solubility of parthenolide in feverfew. From Figure 1 it can be seen that parthenolide dissolves out of feverfew extract more readily under neutral conditions. However, this $\mathrm{pH}$ effect is not very large. Even under the worst condition (a dissolution medium of $\mathrm{pH} 3$ ), the solubility of parthenolide in feverfew is more than $120 \mu \mathrm{g} / \mathrm{mL}$. Generally, a drug is considered to be highly soluble if its highest unit dose can be dissolved in $250 \mathrm{~mL}$ of buffer in the $\mathrm{pH}$ range of 1-8. Our data proved that at least $30 \mathrm{mg}$ parthenolide can be dissolved in $250 \mathrm{~mL}$ medium, which is much more than the maximum daily dose defined by the Canadian feverfew monograph. This observation indicates that the parthenolide in feverfew is highly soluble, and sink conditions are readily approximated.

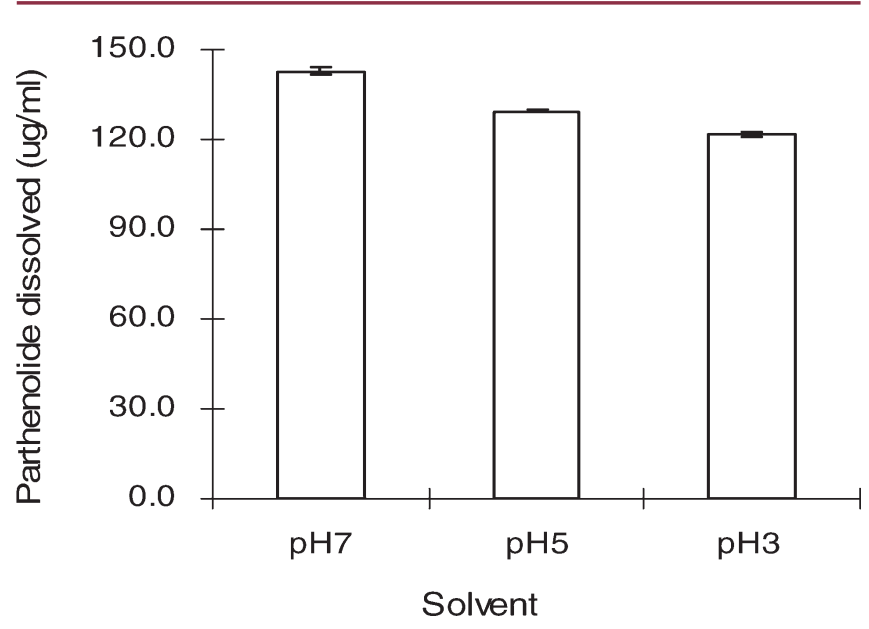

Figure 1.The effect of $\mathrm{pH}$ value on solubility of parthenolide in feverfew. The error bars represent \%RSD $(\mathrm{n}=3)$. 
However, the solubility of parthenolide in feverfew found in this study is not necessarily the intrinsic solubility of pure parthenolide. Even a small amount of impurity can affect the solubility of the pure compound. Here, parthenolide generally accounts for less than $1 \%$ of feverfew. On one hand, the dissolution of other compounds, especially hydrophobic compounds, could affect the polarity of the dissolution medium and thus improve the solubility of parthenolide in the medium. On the other hand, the interaction between parthenolide and other compounds may interfere with the release of parthenolide out of feverfew. Smith and Burford (16) proposed that parthenolide was present at different sites in the feverfew plant matrix. Some are "free" parthenolide on the surface, which are readily dissolved, but in other sites, the parthenolide may be more tightly bound and would require the addition of a modifier to be released. This indicates that even a sink condition does not necessarily guarantee complete dissolution. Figure 2 shows a comparison of the extraction efficiency of different media using $50 \%$ ethanol as control. It can be seen that under the present extraction conditions $(250 \mathrm{mg}$ feverfew containing $\approx 0.2 \%$ parthenolide extracted by $25 \mathrm{~mL}$ medium), the extraction efficiency of $50 \%$ ethanol is significantly higher than that of the aqueous buffers even though the solubility of parthenolide in each extraction medium is sufficient to ensure a complete dissolution. This suggests that the addition of surfactant may be necessary for setting a suitable dissolution condition for feverfew products. As shown in Figure 3, the addition of $0.5 \%$ sodium dodecyl sulfate (SDS) can lead to the same

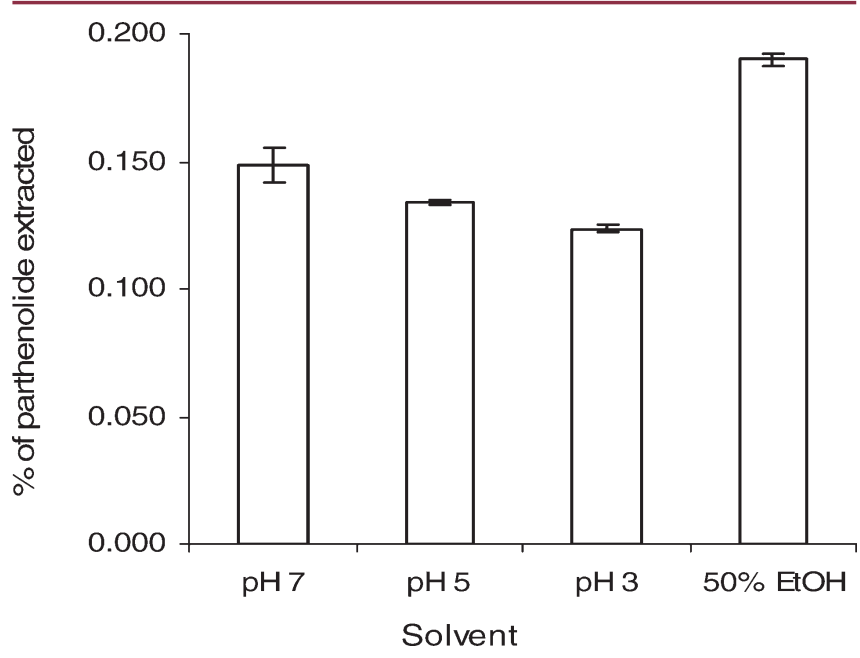

Figure 2. The effect of $\mathrm{pH}$ on extraction efficiency of parthenolide in feverfew. The error bars represent \%RSD $(\mathrm{n}=3)$.

Dissolution Technologies | AUGUST 2007 extraction efficiency as 50\% ethanol/water; higher levels of SDS cannot further improve the extraction efficiency. In Figure 4, the dissolution profile of one commercial feverfew product (P1) in water is compared with or without the addition of SDS. In water, this product could not reach $85 \%$ dissolution even after $2 \mathrm{~h}$. However, with the addition of $0.5 \%$ SDS, more than $85 \%$ dissolution was achieved in $30 \mathrm{~min}$. This indicates that $0.5 \%$ SDS may be a suitable component of media for dissolution testing of feverfew products.

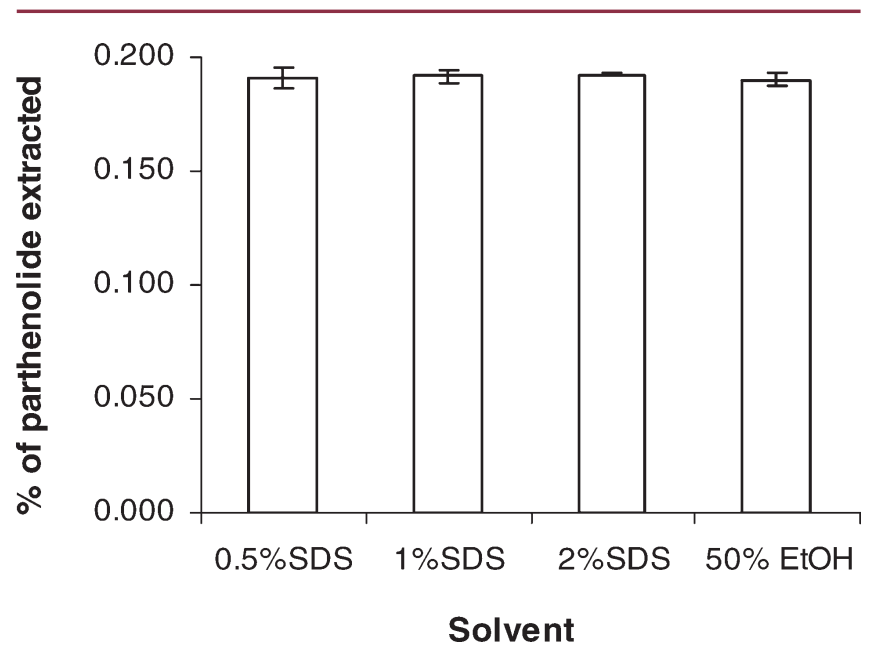

Figure 3. The effect of surfactant concentration on extraction efficiency of parthenolide in feverfew. The error bars represent \%RSD $(\mathrm{n}=3)$.

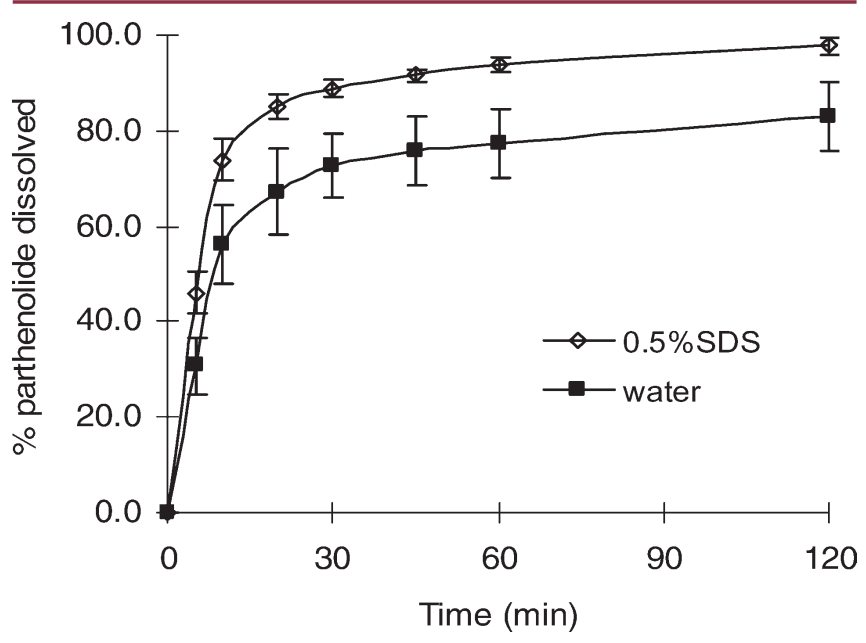

Figure 4. The effect of the addition of $0.5 \%$ sodium dodecyl sulfate (SDS) on the dissolution profile of parthenolide in feverfew. The error bars represent $\% R S D(\mathrm{n}=3)$. 


\section{Dissolution Performance of Several Commercial Feverfew Products}

Based on the data in Table 4, there are substantial differences in the release profiles of the four products, although they can reach more than $85 \%$ dissolution in $1 \mathrm{~h}$. In the first 10 min, one product releases more than $80 \%$, but dissolution from the two other products cannot be detected at all during that time.

Differences in the dissolution profiles could result from disintegration, formulation composition, and hydrodynamic differences among experiments.

Dissolution parameters such as medium volume and stirring rate were identical for all tests, thus minimizing hydrodynamic differences. Disintegration differences may partly explain the differences in dissolution performance. As shown in Table 5 , the order of disintegration time is $\mathrm{P} 1<\mathrm{P} 4<\mathrm{P} 2<\mathrm{P} 3$. Although the order of disintegration matches the order of release rate, the disintegration difference between the most rapid and the slowest among them is less than 4 min, which cannot completely explain the 10-min release lag of P2 and P3.

\section{Table 4. Dissolution of Parthenolide from the Feverfew} Products Studied $(\mathrm{n}=3)$.

Percent of Parthenolide Content Dissolved

\begin{tabular}{lcccc} 
Time $(\mathrm{min})$ & $\mathrm{P} 1$ & $\mathrm{P} 4$ & $\mathrm{P} 3$ & $\mathrm{P} 2$ \\
\hline 5 & $45.9 \pm 4.4$ & $8.6 \pm 4.5$ & $\mathrm{ND}$ & $\mathrm{ND}$ \\
\hline 10 & $73.9 \pm 4.5$ & $36.3 \pm 9.9$ & $\mathrm{ND}$ & $\mathrm{ND}$ \\
\hline 20 & $84.9 \pm 2.5$ & $86.4 \pm 10.0$ & $9.3 \pm 9.5$ & $58.5 \pm 17.0$ \\
\hline 30 & $88.9 \pm 1.8$ & $100.5 \pm 4.7$ & $48.9 \pm 12.5$ & $75.9 \pm 18.2$ \\
\hline 45 & $91.6 \pm 1.1$ & $102.5 \pm 5.3$ & $78.7 \pm 13.6$ & $84.4 \pm 16.7$ \\
\hline 60 & $93.9 \pm 1.7$ & $102.2 \pm 2.7$ & $89.6 \pm 11.3$ & $89.6 \pm 13.0$ \\
\hline 120 & $97.7 \pm 2.0$ & $101.5 \pm 3.5$ & $94.8 \pm 4.0$ & $96.2 \pm 8.3$ \\
\hline
\end{tabular}

ND: None detected

Table 5. Comparison of Disintegration Times of Commercial Feverfew Products.

\begin{tabular}{lc}
\hline Product & $\begin{array}{c}\text { Disintegration Time (min) } \\
\text { Mean } \pm \text { SD }(n=6)\end{array}$ \\
\hline P1 & $5.05 \pm 1.39$ \\
\hline P2 & $6.54 \pm 0.92^{\mathrm{a}}$ \\
\hline P3 & $8.92 \pm 1.86^{\mathrm{a}, \mathrm{b}}$ \\
\hline P4 & $5.22 \pm 0.36^{\mathrm{b}}$ \\
\hline
\end{tabular}

${ }^{a}$ significantly different from P1 $(p<0.05)$

${ }^{\mathrm{b}}$ significantly different from P2 $(p<0.05)$
Excipients in the formulation could influence solubility and wettability of the matrix. De Souza et al. (17) studied the influence of excipients on the dissolution profiles of Maytenus ilicifolia tablets and found that the release from formulations containing lactose occurred much faster than from cellulose formulations, which is attributed to the differences in excipient solubility, tablet hardness, and water uptake capacity. Based on the information in Table 1, apparently P1 and P4 only contained feverfew extract or feverfew powder, while P2 and P4 combined excipient and P4 even contained vitamins and other botanical extract. The interaction between parthenolide and other components in the formulation may be a cause for slower parthenolide release. In addition, the nature of the dry extracts used to manufacture the products could play a very important role in this case. The ratio between "free" and "bound" parthenolide, the composition of inactive components, and the particle size of dry extracts are all factors that possibly could affect the release rate of finished products.

The solubility data presented in this paper, together with information on parthenolide absorption published by Khan et al. (14) (discussed above), suggest that parthenolide is a high solubility-high permeability compound. Since, in this case, absorption is likely to be limited by gastric emptying rather than dissolution rate, an IVIVC is not expected. Accordingly, the utility of a dissolution test for feverfew products would most likely be to help ensure batch-to-batch consistency. The significantly different release profiles we found among feverfew products from different manufacturers indicate that the dissolution method developed here has enough discriminatory power to resolve manufacturing differences and been fully validated.

EMEA proposed that if active components of standardized extracts are known to be highly soluble throughout the physiological $\mathrm{pH}$ range, a disintegration test may substitute for the dissolution test so long as they are formulated as immediate-release products. The existence of a release lag in our study suggests that this substitution needs to be considered case-by-case. The active components of botanicals exist in very complicated environments that may decrease the correlation between disintegration and dissolution. Formulation and manufacturing variables may also adversely affect release characteristics. A release lag has been observed for other botanical products. For example, Westerhoff et al. (18) studied the release performance of St. John's Wort products and found a lag time of about $1 \mathrm{~h}$ for the release of rutin and hyperforin from one of five products studied. Disintegration time was not studied in that paper. However, if the relationship between disintegration and 
dissolution has already been established for a given product, dissolution testing may not always be necessary or may be proposed as a periodic test.

\section{Conclusion}

The commercial feverfew products studied exhibited a wide range in parthenolide content per dosage unit. One product contained no detectable parthenolide. The remaining products tested provided various parthenolide contents and showed large differences between actual parthenolide content and their label claims. Disintegration and dissolution need not necessarily be a major problem for feverfew products because all tested products containing parthenolide were found to disintegrate within 20 min and exhibit more than 85\% dissolution in $1 \mathrm{~h}$. However, dissolution profiles were substantially different among them, with two products exhibiting about a 10-min lag time. Patients should be cautious when they change from one brand to another. Manufacturers should commit to proper quality control procedures that ensure accurate and realistic label claims for content and dose. Formulations should be designed to provide rapid, consistent release characteristics.

\section{References}

1. United States Pharmacopeia and National Formulary USP 30-NF 25; The United States Pharmacopeial Convention, Inc.: Rockville, MD, 2007.

2. Current Good Manufacturing Practice in Manufacturing, Packaging, Labeling, or Holding Operations for Dietary Supplements, Final Rule; U.S. Food and Drug Administration, Office of Regulatory Affairs and Center for Food Safety and Applied Nutrition; U.S. Government Printing Office:Washington, DC, 2007.

3. European Pharmacopoeia, 5th ed.; European Directorate for the Quality of Medicines; Council of Europe: Strasbourg, France, 2005.

4. Committee for Medicinal Products for Human Use (CHMP), Committee for Medicinal Products for Veterinary Use (CVMP). Guideline on Specifications: Test Procedures and Acceptance Criteria for Herbal Substances, Herbal Preparations and Herbal Medicinal Products/Traditional Herbal Medicinal Products. European Medicines Evaluation Agency (EMEA): London, UK, 2005.

5. Fitzpatrick, K. C. Nutraceuticals-a booming industry. In Herbs 2000, Proceedings of the International Herb Conference, Saskatoon, SK, July 18-22, 2000; International Herb Association and Canadian Herb Society: Saskatoon, SK, 2000.

6. Kemper, K. J. Feverfew (Tanacetum parthenium). The Longwood Herbal Task Force and the Center for
Holistic Pediatric Education and Research, 1999. http://www.longwoodherbal.org/feverfew/feverfew. pdf (accessed July 6, 2007).

7. Feverfew. Health Canada, 2006. http://www.hc-sc.gc. ca/dhp-mps/alt_formats/hpfb-dgpsa/pdf/prodnatur/ mono_feverfew-camomille_e.pdf (accessed July 6 , 2007).

8. Abourashed, E. A; Khan, I. A. Determination of parthenolide in selected feverfew products by liquid chromatography. J. AOAC Int. 2000, 83 (4), 789-792.

9. Draves, A. H.;Walker, S. E. Parthenolide content of Canadian commercial feverfew preparations: label claims are misleading in most cases. Pharm. Res. Rev. 2004, 136 (10), 23-30.

10. Nelson, M. H.; Cobb, S. E.; Shelton, J.Variations in parthenolide content and daily dose of feverfew products. Am. J. Health Syst. Pharm. 2002, 59 (16), 1527-1531.

11. Jin, P.; Madieh, S.; Augsburger, L. L. Selected Physical and Chemical Properties of Feverfew (Tanacetum parthenium) Extracts Important for Formulated Product Quality and Performance. AAPS PharmSci, submitted for publication, 2007.

12. Dissolution Testing of Immediate Release Solid Oral Dosage Forms; Guidance for Industry; U.S. Department of Health and Human Services, Food and Drug Administration, U.S. Government Printing Office: Washington, DC, August 1997.

13. Amidon, G. L.; Lennernas, H.; Shah, V. P.; Crison, J.R. A theoretical basis for a biopharmaceutic drug classification:The correlation of in vitro drug product dissolution and in vivo bioavailability. Pharm. Res. 1995, 12,413-420.

14. Khan, S. I.; Abourashed, E. A.; Khan, I. A.; Walker, L. A. Transport of parthenolide across human intestinal cells (Caco-2). Planta Med. 2003, 69 (11), 1009-1012.

15. Hanson, R.; Gray, V. Handbook of Dissolution Testing, 3rd ed.; Dissolution Technologies, Inc.: Hockessin, DE, 2004.

16. Smith, R. M.; Burford, M. Supercritical fluid extraction and gas chromatographic determination of the sesquiterpene lacton parthenolide in the medicinal herb feverfew.J. Chromatogr. 1992, 627, 255-261.

17. de Souza, T. P.; Bassani, V. L.; Gonzalez Ortega, G.; dalla Costa, T.C.; Petrovick, P. R. Influence of adjuvants on the dissolution profile of tablets containing high doses of spray-dried extract of Maytenus ilicifolia. Pharmazie 2001, 56 (9), 730-733.

18. Westerhoff, K.; Kaunzinger, A., Wurglics, M.; Dressman, J.; Schubert-Zsilavecz, M. Biorelevant dissolution testing of St John's wort products. J. Pharm. Pharmacol. 2002, 54 (12), 1615-1621. 